\title{
Commentary: Is transfusion as simple as Goldilocks makes it? "For every complex problem there is an answer that is clear, simple, and wrong"**
}

In this issue of the Journal, an Invited Expert Opinion addresses an article in The New England Journal by Mazer and colleagues ${ }^{1}$ that presented the 6-month follow up from a truly unique study called the "TRICS III randomized trial." The TRICS III trial evaluated outcomes in moderate- to high-risk cardiac surgical patients treated with a restrictive transfusion threshold (hemoglobin $<7.5 \mathrm{~g} / \mathrm{dL})$ compared with those transfused with a liberal threshold $(<9.5 \mathrm{~g} / \mathrm{dL})$. This trial was one of several that used hemoglobin levels as a metric for transfusion decisions during and after cardiac operations. Our Journal editors thought that the article ${ }^{1}$ was an impactful study that affects a broad spectrum of cardiac surgical patients, so much so that they thought that Editorial comments were necessary to place this study in perspective. One of these editorial comments was written by Hensley and co-authors $^{2}$ and provides some thoughtful, critical comments about the balance between overuse and, although not well defined, underuse of transfusion.

As Hensley and colleagues ${ }^{2}$ point out, decision making related to perioperative blood transfusion is by no means an exact science. Indeed, the fact that decisions about transfusion hinge on only hemoglobin "triggers" may be one of the most unscientific things that cardiac surgeons do! There are rare scenarios in the practice of cardiac surgery that rely purely on a single number without consideration of physiology, anatomy, patient-specific factors, and untold other objective and clinical information. This peculiarity of blood transfusion opens the door for clinical research and studies that provide new evidence-based indicators for transfusion. A quote from Hensley and colleagues ${ }^{2}$ sets the stage for things that may lie in the future of blood transfusion: "We currently lack technology to detect, in real-time,

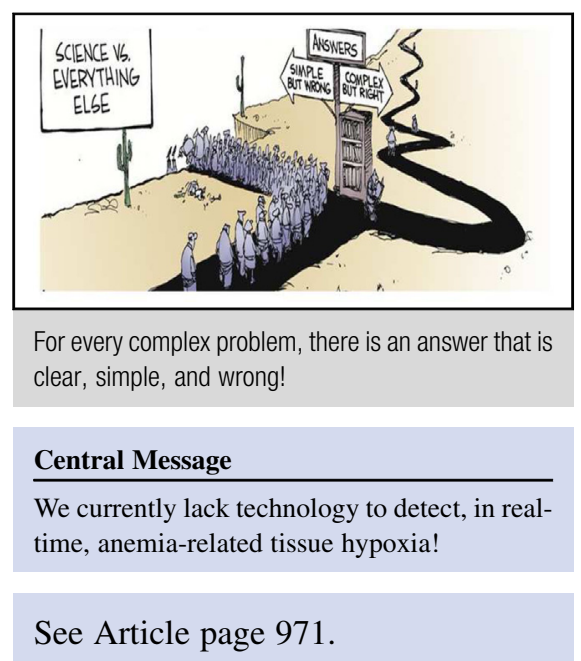

anemia-related tissue hypoxia, and the influence of the perioperative milieu and patient specific factors on the overlay of acute anemia. While we currently are unable to get it 'just right' for each individual patient, results from trials such as Mazer and colleagues provide us data that will move us further away from overuse and allow us to contribute to reductions in overall healthcare waste."

This quote, in different versions, has been stated repeatedly over the last few decades. Many authors recognized the knowledge gap, but few have devised ways to expand the knowledge base required to make evidence-based transfusion decisions on anything more than hemoglobin triggers.

The Invited Expert Opinion by Hensley and colleagues ${ }^{2}$ raises questions about "useful" and "wasteful" transfusion practices. It is presumptive to talk about overuse, underuse, and quality defects in transfusion practices without better understanding of detailed transfusion indicators and physiologic endpoints of transfusion. It is entirely possible that some patients who receive "overuse" transfusions may in fact benefit from improved oxygen delivery or better tissue level oxygenation independent of hemoglobin levels. Without tissue level measurements of red cell-related oxygenation, it is difficult if not impossible to adequately characterize adequate transfusion practices. It is worth printing a quote from one of the authors that sums up this quandary. 
"Absence of 'evidence' cannot and should not be used to suggest that the data we do have are definitive or adequate to guide transfusion." 3

Colleen $\mathrm{Koch}^{4}$ summed up this dilemma in a thoughtful article about transfusion triggers. She suggested that a widely used transfusion trigger of $7 \mathrm{~g} / \mathrm{dL}$ seems like a simple solution to the very complex problem of when to transfuse patients. However, she was quick to paraphrase a quote attributed to H. L. Mencken: "For every complex problem there is an answer that is clear, simple, and wrong." "There is little doubt that the conventional transfusion triggers lack the physiologic and evidence-based imperatives to justify universal adoption of strict hemoglobin triggers for transfusion.

So what can be done about this evidence deficit? The TRICS III trial is a good start, and it challenges the increased operative mortality associated with restrictive transfusion found in earlier trials (eg, the TITRe2 trial). ${ }^{5}$ The TRICS III trial provides data to support the current thought that use of a restrictive packed red cell transfusion trigger in moderate- to high-risk patients undergoing cardiac operations is not associated with harm. In addition, the use of restrictive transfusion practice leads to resource reduction without apparent injury.

An intriguing thought that surfaces in discussions about transfusion benefits deals with speculation about better indicators of transfusion needs that rely on measurable physiologic cellular and molecular parameters that both predict reduced injury and increased benefit. There are suggestions that measuring the ratio of splanchnic to cerebral oxygenation may indicate the need for transfusion to support vital tissue oxygenation, especially in children. ${ }^{6,7}$ Further, peripheral fractional oxygen extraction as a measure of tissue oxygenation may be a better guide to the need for blood transfusion and an improved guide to tissue oxygen use benefit compared with simple transfusion hemoglobin triggers. ${ }^{8,9}$ Measures of erythrocyte flexibility may assess potential for microvascular perfusion and oxygenation to a greater extent than simple hemoglobin measurements. ${ }^{10}$ There is an external monitor of cellular oxygen metabolism that has shown promise for real-time measurement of mitochondrial oxygen tension. ${ }^{11}$ This monitor has the acronym of Cellular Oxygen METabolism (COMET) and needs to be tested as a monitor of microvascular flow in cardiac surgical patients. There are other potential monitoring tools that may have applications in cardiac surgical patients. There is an obvious need for improved measurement of both indications for and benefits of transfusion. It is surprising that the Goldilocks principle is the best we can do to guide transfusion needs.

The question that remains is the Goldilocks tale appropriate for deciding about perioperative transfusion? What begs the question is not whether finding the right or "sweet spot" as described in the Goldilocks tale is best practice, but the real issue is whether transfusion at that "sweet spot" is best therapy for the patients' anemia. To find this alluring spot where no harm is apparent, we still must quantitate the benefit. An even greater challenge resides beyond red cell therapy. There is not near the evidence base to inform decisions about platelets, plasma, and other fractionated blood components in cardiac and noncardiac operations. Further research needs to focus on physiologic evidencebased transfusion indicators and when other competing therapies are warranted. Until these future studies appear, surgeons are stuck with the evidence from use of transfusion triggers.

\section{References}

1. Mazer CD, Whitlock RP, Fergusson DA, Hall J, Belley-Cote E, Connolly K, et al. Restrictive or liberal red-cell transfusion for cardiac surgery. N Engl J Med. 2017; 377:2133-44.

2. Hensley NB, Brown CH, Frank SM, Koch CG. The Goldilocks principle and perioperative red blood cell transfusion: overuse, underuse, getting it just right J Thorac Cardiovasc Surg. 2020;159:971-3.

3. Shander A, Ferraris VA. More or less? The Goldilocks principle as it applies to red cell transfusions. Br J Anaesth. 2017;118:816-9.

4. Koch CG. Tolerating anemia: taking aim at the right target before pulling the transfusion trigger. Transfusion. 2014;54:2595-7.

5. Murphy GJ, Pike K, Rogers CA, Argent A, Carson JL, Valentine SL, et al. Liberal or restrictive transfusion after cardiac surgery. N Engl J Med. 2015;372: 997-1008.

6. Bailey SM, Hendricks-Munoz KD, Mally P. Splanchnic-cerebral oxygenation ratio as a marker of preterm infant blood transfusion needs. Transfusion. 2012;52: 252-60.

7. Doctor A, Cholette JM, Remy KE, Argent A, Carson JL, Valentine S, et al. Recommendations on RBC transfusion in general critically ill children based on hemoglobin and/or physiologic thresholds from the pediatric critical care transfusion and anemia expertise initiative. Pediatr Crit Care Med. 2018;19: S98-113.

8. Wardle SP, Weindling AM. Peripheral fractional oxygen extraction and other measures of tissue oxygenation to guide blood transfusions in preterm infants. Semin Perinatol. 2001;25:60-4.

9. Hartmann M, Montgomery A, Jonsson K, Haglund U. Tissue oxygenation in hemorrhagic shock measured as transcutaneous oxygen tension, subcutaneous oxygen tension, and gastrointestinal intramucosal $\mathrm{pH}$ in pigs. Crit Care Med. 1991;19:205-10.

10. Cabrales P. Effects of erythrocyte flexibility on microvascular perfusion and oxygenation during acute anemia. Am J Physiol Heart Circ Physiol. 2007;293: H1206-15.

11. Ubbink R, Bettink MAW, Janse R, Harms FA, Johannes T, Münker FM, et al. A monitor for Cellular Oxygen METabolism (COMET): monitoring tissue oxygenation at the mitochondrial level. J Clin Monit Comput. 2017;31:1143-50. 